\title{
Revascularização do miocárdio no paciente octogenário: 15 anos de observação
}

José Carlos R. IGLÉZIAS*, Luís Alberto DALLAN*, Sérgio Ferreira de OLIVEIRA*, José Antônio F. RAMIRES*, Sérgio Almeida de OLIVEIRA*, Geraldo VERGINELLI*, Adib D. JATENE*

RBCCV 44205-219

IGLÉZIAS, J. C. R.; DALLAN, L. A.; OLIVEIRA, S. F.; RAMIRES, J. A. F.; OLIVEIRA, S. A.; VERGINELLI, G.; JATENE, A. D. - Revascularização do miocárdio no paciente octogenário: 15 anos de observação. Rev. Bras. Cir. Cardiovasc., 8 (3): 237-240, 1993.

RESUMO: Como objetivo de avaliar a mortalidade hospitalar atual e comparar a evolução da revascularização convencional do miocárdio nos pacientes octogenários, tratados evolutivamente de Janeiro/1978 a Janeiro/ 1993, no InCór, foram analisados, retrospectivamente, todos os pacientes operados no período. Dos 47 pacientes, $35(74,46 \%)$ eram do sexo masculino e $12(25,53 \%)$ do sexo feminino. A média de idade foi igual a 81,85 (80 a 88) anos. A indicação operatória ocorreu devido ao quadro de angina instável em $29(61,70 \%)$ pacientes, angina estável em $17(36,17 \%)$ pacientes e em $1(2,12 \%)$ paciente devido a dissecção de placa de ateroma quando da realização de angioplastia. As operações foram realizadas em caráter eletivo em 33 $(70,21 \%)$ pacientes, em caráter de urgência em $10(21,27 \%)$ pacientes e em caráter emergencial nos outros $4(8,51 \%)$. Os condutos utilizados foram a veia safena autogêna em $41(87,23 \%)$ pacientes e a artéria torácica interna pediculada em $6(12,76 \%)$. A mortalidade hospitalar foi de $8,5 \%$ e as causas de óbito foram: encefalopatia anóxica, insuficiência respiratória, hemorragia digestiva e choque cardiogênico secundário a infarto agudo do miocárdio. O tempo médio de seguimento foi de 17,6 (1 a 75) meses. As causas de óbito tempo-relacionadas foram: neoplasias em $3(27,27 \%)$ pacientes, infeç̧ăo em $3(27,27 \%)$ pacientes, acidente vascular cerebral em $2(18,18 \%)$ pacientes, trombose mesentérica em $1(9,09 \%)$ paciente, síndrome depressiva em $1(9,09 \%)$ paciente e choque cardiogênico em $1(9,09 \%)$. Analisando a sobrevida tempo-relacionada, observamos que nos 3 últimos períodos: 91,92 e Janeiro/93 a mortalidade caiu para zero. A grande maioria dos pacientes estava livre de angina e de sinais e/ou sintomas de insuficiência cardiaca. Concluímos que a revascularizaçāo convencional do miocárdio representa boa alternativa terapêutica para o paciente octogenário portador de doença arterial coronária, não só devido ao risco operatório decrescente, como também à redução/abolição dos sintomas, melhorando a qualidade e/ou a expectativa de vida.

DESCRITORES: miocárdio, revacularização, pacientes idosos.

\section{INTRODUÇÃO}

O número de pacientes idosos submetidos a revascularização do miocárdio ${ }^{6}$ continua crescendo não somente pelo aumento de idosos na população com doença cardíaca sintomática, mas porque a revascularização do miocárdio parece, genericamente, ser benéfica ao idoso. O envelhecimento está associado com a perda de reservas fisiológicas que afetam orgãos de diferentes sistemas em graus variados, de maneira que o risco de complicaçōes cardiopulmonares, renais, neurológicas podem aumentar com o avançar da idade. A liberalização da indicação operatória no idoso pode determinar o aparecimento de maior número de intercorrências cirúrgicas: daí o melhor conhecimento dos riscos de morbidade relacionados ao coração, ou não, para que possamos aprimorar a indicação operatória.

Trabalho realizado no Instituto do Coração do Hospital das Clínicas da Faculdade de Medicina da Universidade de São Paulo. São Paulo, SP, Brasil. Recebido para publicação em 30 de agosto de 1993.

- Do Instituto do Coração do Hospital das Clinicas da Faculdade de Medicina da Universidade de São Paulo.

Endereço para separatas: José Carlos Iglézias. Av. Dr. Enéas de Carvalho Aguiar, 44. Divisão Cirúrgica, 05403 - São Paulo, SP, Brasil. 
IGLÉZIAS, J. C. R.; DALLAN, L. A.; OLIVEIRA, S. F.; RAMIRES, J. A. F.; OLIVEIRA, S. A.; VERGINELLI, G.; JATENE, A. D. Revascularização do miocárdio no paciente octogenário: 15 anos de observação. Rev. Bras. Cir. Cardiovasc., 8(3):237-240, 1993.

Ainda que muitos estudos retrospectivos sugiram os idosos mais suscetíveis a complicações após a revascularização do miocárdio e a idade ser fator de risco para as disfunções neurológicas no pósoperatório, a maioria dos estudos prospectivos, analisando danos neurológicos após cirúrgia cardiaca, não considera a idade como um fator de risco, e quando existe, o número de pacientes estudados é pequeno.

Embora avanços na técnica cirúrgica, anestesia, cuidados intensivos, técnica de perfusão tenham reduzido substancialmente a morbidade e a mortalidade relacionadas às operações que requerem circulação extracorpórea, complicações transitórias ou permanentes envolvendo o sistema nervoso central continuam ocorrendo e, talvez, com freqüência crescente, a despeito de melhoria no estado funcional cardiovascular.

Mesmo sabendo que os idosos estão sujeitos a maior número de eventos não cardíacos relacionados ao pós operatório, elaboramos este estudo visando determinar a mortalidade hospitalar atual e a evolução comparativa, por períodos, na Instituição, nos pacientes octogenários.

\section{CASUÍSTICA, MÉTODOS E RESULTADOS}

Para avaliar o risco operatório atual do tratamento cirúrgico da insuficiência no paciente octogenário e estabelecer a evolução comparativa, por períodos, foram analisados todos os pacientes operados, no InCór, de Janeiro/78 a Janeiro/93.

Nenhum método estatístico específico foi aplicado a esta casuística. Trata-se de uma análise retrospectiva, para fins comparativos.

Foram analisados retrospectivamente todos os pacientes octogenários, portadores de doença arterial coronária, e que se submeteram a revascularização convencional do miocárdio no InCór, no período compreendido entre Janeiro/78 e Janeiro/ 93. Constituíram um total de 47 pacientes, sendo que $35(74,46 \%)$ eram do sexo masculino e 12 $(25,33 \%)$ do sexo feminino. A média de idade foi de 81,85 (80 a 88) anos. A indicação operatória ocorreu devido ao quadro de angina instável em 29 $(61,70 \%)$ pacientes, angina estável em $17(36,17 \%)$ pacientes e em $10(2,12 \%)$ paciente devido a dissecção de placa de ateroma quando da realização de angioplastia coronária percutânea. A operação foi realizada em caráter eletivo em 33 (70,21\%) pacientes, em caráter de urgência em $10(21,27 \%)$ pacientes e em caráter emergencial em $4(8,51 \%)$ pacientes.

Todos foram submetidos a toracotomia media- na, circulação extracorpórea e hipotermia moderada. Oconduto mais utilizado para a realização dos enxertos foi a veia safena autógena, em 41 (87,23\%) Pacientes; 6 (12,76\%) pacientes receberam enxerto pediculado de artéria torácica interna isoladamente ou em combinação com enxertos venosos.

A mortalidade hospitalar global foi igual a $8,5 \%$ (4/47) e suas causas foram: encefalopatia anóxica em $1(25 \%)$ paciente, hemorragia digestiva alta em $1(25 \%)$ paciente, insuficiência respiratória em 1 (25\%) paciente e choque cardiogênico, secundário a infarto agudo do miocárdio, em 1 (25\%) paciente.

A sobrevida tempo-relacionada teve média de seguimento igual a 17,6 (1 a 75) meses. As causas de óbito tardio foram: neoplasia em $3(27,27 \%)$ pacientes, infecção em $3(27,27 \%)$ pacientes, acidente vascular cerebral em $2(18,18 \%)$ pacientes, trombose mesentérica em $1(9,09 \%)$ paciente, síndrome depressiva em $1(9,09 \%)$ paciente e choque cardiogênico, secundário a infarto agudo do miocárdio, em $1(9,09 \%)$ paciente

A grande maioria dos pacientes estava livre de sintomas relacionados com a insuficiência coronária e insuficiência cardiaca, durante o seguimento tardio.

\section{COMENTÁRIOS}

Observou-se, em nossa Instituição, uma diminuição na mortalidade hospitalar global, no período analisado, neste segmento populacional. Em 1990 ela foi de $13,33 \%$ e no momento está em $8,5 \%$. Através da análise da curva de sobrevida tempo-relacionada, podemos observar que, nos períodos: 1991, 1992 e Janeiro/1993, a mortalidade caiu para zero.

Estamos de acordo com MULLANY et alii ${ }^{8}$, quando, ao analisar 159 pacientes com idade igual ou superior a 80 anos, submetidos a revascularização do miocárdio, na Mayo Clinic, desde 1977, concluíram que a operação em pacientes nesta faixa etária, embora associada com risco operatório aumentado, propicia excelente alívio dos sintomas, boa sobrevida de 5 anos e que não podem ser desconsiderados para a revascularização do miocárdio somente pela idade.

Em nossa casuística não há concordância com LOOP et alii ${ }^{7}$, que analisam 5070 pacientes com idade igual ou superior a 65 anos, subdivididos em dois grupos: com idade variando entre 65 e 74 anos e o outro incluindo os com mais de 75 anos e comparam aos pacientes mais jovens, e afirmam que, com o avançar da idade, existe grande prevalência de mulheres. Observamos, nos pacientes octogenários da casuística em questão uma proporção aproximada de 3:1 com predomínio para o sexo masculino. 
IGLÉZIAS, J. C. R.; DALLAN, L. A.; OLIVEIRA, S. F.; RAMIRES, J. A. F.; OLIVEIRA, S. A.; VERGINELLI, G.; JATENE, A. D. Revascularização do miocárdio no paciente octogenário: 15 anos de observação. Rev. Bras. Cir. Cardiovasc., 8 (3):237-240, 1993.

Concordamos com COSGROVE et alii ${ }^{1,2}$, quando afirmam, ao analisar 24.672 pacientes no período compreendido entre 1970 e 1982, divididos em quatro subgrupos, que a idade avançada constitui um fator incrementador de risco para morbidade e mortalidade. Isto porém não contra-indica a operação uma vez que as maiores causas de mortalidade hospitalar e tardia, assim como em nossa casuística, não são cardíacas. Existe correspondência ainda entre as duas casuísticas quanto à afirmação de que tanto a morbidade como a mortalidade têm decrescido significativamente, a despeito do aumento dos fatores de risco; a morte de causa cardíaca vem diminuindo e sendo substituída pela falência de outros sistemas.

Estamos de acordo com EDMUNDS Jr. et alii ${ }^{3}$, quando afirmam, após análise de 100 pacientes octogenários consecutivos, submetidos a revascularização convencional do miocárdio no período compreendido entre julho/1976 e maio/1987, na Universidade da Pensilvânia: nos pacientes idosos acometidos por sintomas intratáveis, a operação pode representar uma opção terapêutica eficaz.

A mortalidade hospitalar global do período, na Instituição $(8,5 \%)^{6}$, foi semelhante à encontrada por $\mathrm{HE}$ et alii ${ }^{5}(8,86 \%)$ ao analisarem 1399 pacientes idosos submetidos a revascularização do miocárdio no Medical City Dallas Hospital, Texas, no período compreendido entre janeiro/1986 e junho/ 1993. Segundo sua casuística a utilização da artéria toracica interna esquerda pediculada está associada a menor mortalidade hospitalar, mesmo se comparada a utilização da veia safena autógena. Em nossa casuística os condutos usados preferencialmente foram os segmentos venosos.

GALBUT et alii ${ }^{4}$ encontraram menor mortalidade hospitalar com o uso bilateral da artéria torácica interna. A sobrevida imediata para os pacientes que receberam um enxerto de artéria torácica interna foi de $96,4 \%$ e para aqueles que receberam 2 enxertos pediculados foi de $97,7 \%$. Em nossa casuística, até a data analisada, o enxerto duplo de artéria torácica interna não havia sido realizado.

Baseados na casuística concluímos que a revascularização convencional do miocárdio representa boa alternativa terapêtica para o paciente octogenário portador de doença arterial coronária, não só devido ao risco operatório decrescente como também à redução/abolição dos sintomas, aumentando a expectativa de vida.

RBCCV $44205-219$

IGLÉZIAS, J. C. R.; DALLAN, L. A.; OLIVEIRA, S. F.; RAMIRES, J. A. F.; OLIVEIRA, S. A.; VERGINELLI, G.; JATENE, A. D. - Myocardial revascularization in the octogenarian: fifteen years of observation. Rev. Bras. Cir. Cardiovasc., 8(3): 237-240, 1993.

ABSTRACT: This is a retrospective study where 47 patients submitted to the conventional revascularization of the myocardium were analysed at InCór in a period covering January/78 to January/93; 47 patients were analysed. Thirty-tive $(74.46 \%)$ were male and $12(25.33 \%)$ female. The mean age was $81.85(80-80)$ years. The operatory indication was due to unstable angina in $29(61.70 \%)$, stable angina in $17(36.17 \%)$ and dissection of the atheromatous plaque during angioplasty in $1(2.12 \%)$. The operation was carried out eletively in $33(70.21 \%)$, in caracter of urgency in $10(21.27 \%)$ and in emergency in $4(8.51 \%)$. All of them were operated on through median thoracotomy with extracorporeal circulation and moderate hypotermia. The saphenous vein was used as a graft in $41(87.23 \%)$ patients and the internal thoracic artery in $6(12.76 \%)$. Hospital mortality was $8.5 \%$ and the mean follow-up time was 17,6 months. From among the early and late deaths only one of each group was related to cardiopathy. Analysis of the cases revealed that in the period of 1991,1992 and January/1993, hospital mortality fell down to zero. Surgical conventional revascularization of the myocardium represents a good alternative for the octogenarian patient, not only due to the present operative low risk, but as well as the reduction or elimination of the symptoms during the late follow-up.

DESCRIPTORS: myocardial revascularizations, octogenarians.

\section{REFERÊNCIAS BIBLIOGRÁFICAS}

1 COSGROVE, D. M.; LOOP, F. D.; LYTLE, B. W. Predictors of reoperation after myocardial revascularization. J. Thorac. Cardiovasc. Surg., 92: 811$821,1986$.
2 COSGROVE, D. M.; LOOP, F. D.; LYTLE, B. W.; BAILLOT, R.; GILL, C. C.; GOLDING, L. A. R.; TAYLOR, P. C.; GOORMASTIC, M. - Primary myocardial revascularization. Trends in surgical mortality. J. Thorac. Cardiovasc. Surg., 88: 673$684,1984$. 
IGLÉZIAS, J. C. R.; DALLAN, L. A.; OLIVEIRA, S. F.; RAMIRES, J. A. F.; OLIVEIRA, S. A.; VERGINELLI, G.; JATENE, A. D. Revascularização do miocárdio no paciente octogenário: 15 anos de observação. Rev. Bras. Cir. Cardiovasc., 8 (3):237-240, 1993.

3 EDMUNDS Jr., L. H.; STEPHENSON, L. W.; EDIE, R. N.; RATCLIFF, M. B. - Open heart surgery in octagenarians. N. Engl. J. Med., 319: 131-136, 1988.

4 GALBUT, D. L.; TRAAD, E. A.; DORMAN, M. J.; DEWITT, P. L.; LARSEN, P. B.; KURLAN SKY, P. A. CARRILLO, R. G.; GENTSCH, T. O. EBRA, G. Coronary bypass grafting in elderly. J. Thorac. Cardiovasc. Surg., 106: 128-136, 1993.

5 HE, G. W.; ACUFF, T. E.; RYAN, W. H.; BOWMAN, R. T.; DOUTHIT, M. B.; MACK, M. J. - Determinants of operative mortality in elderly patients undergoing coronary artery bypass grafting. J. Thorac. Cardiovasc. Surg., 108: 73-81, 1994.

6 IGÉZIAS, J. C. R.; LOURENÇĀO Jr., A.; DALLAN, L. A.; GUTIERRES, P. S.; RAMIRES, J. A. F.; STOLF,
N. A. G.; OLIVEIRA, S. A.; VERGINELLI, G.; JATENE, A. D. - Octogenários: resultados de 3659 necropsias (12 anos de observação). Rev. Bras. Cir. Cardiovasc., 8: 39-43, 1993.

7 LOOP, F. D.; BRUCE, W. L.; COSGROVE, D. M.; GOORMASTIC, M.; TAYLOR, P. C.; GOLD ING. L. A. R.; STEWART, R. W.; GILL, C. C. - Coronary artery bypass graft surgery in the elderly. Cleveland Clin. J. Med., 55: 23-34, 1988.

8 MULLANY, C. J.; DARLING, G. E.; PLUTH, J. R.; ORSZULAC, T. A.; SCHAFF, H. V.; ILSTRUP, D. M.; GERSH, B. J. - Early and late results after isolated coronary artery bypass surgery in 159 patients aged 80 years and older. Circulation, 82 (Supl. 4): 229 236, 1990. 